\title{
Ultrasonography-assisted flexible ureteroscope for the treatment of parapelvic renal cysts: A comparison between the 1470-nm diode laser and the holmium laser
}

\author{
YU CHEN $^{1,2}$, RONGJIANG WANG ${ }^{2}$, XUFENG SHEN $^{2}$, JIANER TANG $^{2}$, JUNWEN SHEN $^{2}$, \\ ZHIHAI FANG ${ }^{2}$, ZHANQIN SHI ${ }^{2}$ and XIAODONG JIN ${ }^{1}$ \\ ${ }^{1}$ Department of Urology, The First Affiliated Hospital of Zhejiang University School of Medicine; \\ ${ }^{2}$ Department of Urology, The First Affiliated Hospital of Huzhou Teacher's University, \\ The First People's Hospital of Huzhou City, Huzhou, Zhejiang 313100, P.R. China
}

Received March 05, 2020; Accepted October 30, 2020

DOI: $10.3892 /$ etm.2020.9603

\begin{abstract}
The present study aimed to compare the efficacy and safety of a flexible ureteroscopic holmium laser incision with flexible ureteroscopic 1470-nm diode laser incision for the treatment of parapelvic renal cysts. The current study collected and analysing the clinical data of 90 independent renal cysts cases retrospectively, including 43 renal cysts cases that received holmium laser surgery (holmium laser group) and 47 renal cysts cases that received 1470-nm diode laser surgery (1470-nm diode laser group). Each group was divided into a thin-walled cyst subgroup and thick-walled cyst subgroup according to cyst wall thickness. Intracapsular hematoma was significantly lower in the 1470-nm diode laser group compared with the holmium laser group ( $0 / 47$ vs. 4/43; $\mathrm{P}=0.048)$. The incision diameter in the 1470-nm diode laser group was significantly larger than the holmium laser group in the thick-walled parapelvic renal cysts subgroup [1.70 $(1.50,1.90)$ vs. $1.30(1.25,1.70) \mathrm{cm} ; \mathrm{P}=0.007]$. The renal cystic diameter of the two groups was markedly reduced one and six months after surgery. The difference was non-significant in the diameter of the renal cyst in the thin-walled cysts subgroups between the two laser groups 6 months after surgery $(1.01 \pm 0.38$ vs. $1.03 \pm 0.53 \mathrm{~cm} ; \mathrm{P}=0.454)$. However, the diameter of the renal cyst in the thick-walled cysts subgroup treated with the 1470-nm diode laser was significantly lower compared with the thick-walled cysts subgroup treated with the holmium laser 6 months after surgery $(1.21 \pm 0.57$ vs. $1.88 \pm 0.94 \mathrm{~cm} ; \mathrm{P}=0.002)$. The results demonstrated that the use of a $1470-\mathrm{nm}$ diode laser or holmium laser surgery under a flexible ureteroscope is a safe and effective treatment for parapelvic renal cysts.
\end{abstract}

Correspondence to: Dr Xiaodong Jin, Department of Urology, The First Affiliated Hospital of Zhejiang University School of Medicine, 79 Qinchun Road, Hangzhou, Zhejiang 310003, P.R. China E-mail: xiaodong-jin@zju.edu.cn

Key words: flexible ureteroscope, 1470-nm diode laser, holmium laser, parapelvic renal cysts
For thick-walled parapelvic renal cysts, the 1470-nm diode laser appears to exhibit a lower postoperative recurrence rate and better long-term postoperative effects due to its improved haemostatic effect and larger intraoperative incision diameter.

\section{Introduction}

Parapelvic renal cysts are renal cystic lesions that most often occur adjacent to the renal collecting system (1). The majority of parapelvic renal cysts are asymptomatic and found incidentally, but occasionally these lesions are discovered because of pain, occult blood in the urine, pyelonephritis, hypertension or renal dysfunction. It is generally accepted that the treatment of parapelvic cysts is more difficult than that of simple renal cysts (2). Traditional treatments of parapelvic renal cysts include a period of observation, puncture, drainage of the percutaneous renal cyst under ultrasonography and laparoscopic renal cyst decortication (3). It has been discovered that laparoscopic decortication of parapelvic cysts requires advanced surgical skills because of the complexity of the cysts and their proximity to the renal hilar structures and the collecting system. The percutaneous approach is a safe and effective method for treating peripherally located simple renal cysts but carries a risk of damage to renal hilar structures and the collecting system for parapelvic cysts (2). With the development of technology, the advantages of flexible ureteroscopy have become more apparent in the treatment of parapelvic cysts $(4,5)$. Parapelvic cysts are characterized by their complexity and proximity to the renal hilar structures $(2,6)$. Endoscopic surgery avoids the risk of damage to the renal hilar structures, and complicated surgical skills are not required to perform laparoscopic approaches. Novel technology devices for laser vaporization have been recently reported, and these devices have been used in prostatectomy $(7,8)$. Technological development in laser vaporization has resulted in higher-powered lasers, such as the 1470-nm diode laser. Water and haemoglobin easily absorb the 1470-nm diode laser, and it is thought to possess good haemostatic properties and high tissue vaporization (7). The present study applied laser devices at 1470-nm diode wavelengths to patients with parapelvic renal cysts to evaluate the potential 
role of 1470-nm diode laser vaporization of parapelvic renal cysts. The results revealed that this may be used as a novel alternative choice for the treatment of parapelvic renal cysts.

\section{Materials and methods}

Patients. A total of 65 patients charged into the First Affiliated Hospital of Huzhou Teacher's University (Huzhou, China) from June 2016 to December 2018 (37 males and 28 females; $55.6 \pm 13.6$ years old) with parapelvic renal cysts were included in the current retrospective study from January 2016 to June 2019. All patients underwent renal enhanced CT and multi-planar reconstruction CT urography before surgery to observe the relationship between renal cysts and the renal pelvis. If the renal cysts were closely adjacent to the renal pelvis in a horizontal position, the coronal position and the sagittal position, and the adjacent width was $>10 \mathrm{~mm}$ in at least one position, the cyst was defined as a parapelvic renal cyst. A total of 9 renal cysts were adjacent to the renal collecting system and protruded from the surface of the kidney. The inclusion criteria used for including patient data was as follows: i) Recurrent or persistent pain without improvement following conservative treatment; ii) repeated urinary tract infection combined with or without renal calculi, without an increase after conservative treatment; iii) renal cysts suggested on CT imaging, with normal renal function or mild-to-moderate lesions (preoperative blood pressure was controlled at $<160 / 100 \mathrm{mmHg}$, Fasting blood glucose was controlled at $<10 \mathrm{mmol} / 1$ and postprandial blood glucose at $<12 \mathrm{mmol} / \mathrm{l})$; iv) renal cystic diameter $>4 \mathrm{~cm}$; v) blood pressure and glucose controlled within the normal range; and vi) Bosniak classification of $\mathrm{CT}$ imaging grades I and II (9). The exclusion criteria was as follows: i) History of the renal tumour and nephrapostasis; ii severe cardiac, hepatic, pulmonary and brain dysfunction, without tolerance to general anaesthesia; iii) Bosniak classification of CT imaging grades III and IV (9); or iv) a polycystic kidney. All clinical experiments and laboratory research were performed according to protocols that were approved by the ethical committee the First Affiliated Hospital of Huzhou Teacher's University, and each patient participated after providing written informed consent.

Definition of thin-walled and thick-walled parapelvic renal cysts. Thin-walled parapelvic renal cysts were considered when there was no obvious boundary between the renal cyst wall and the mucosa of the renal pelvis and calyx. The mucosa of the renal pelvis and calyx was commonly blue translucent or transparent, and the diameter of the blue translucent or transparent area was $>1 \mathrm{~cm}$ under the flexible ureteroscope (Fig. 1A-C). Thick-walled parapelvic renal cysts were considered when a gap existed between the renal cyst wall and the mucosa of the renal pelvis and calyx. The blue translucent or transparent area on the mucosa of the renal pelvis and calyx was commonly non-obvious under the flexible ureteroscope, or the diameter of the blue translucent or transparent area was $<1 \mathrm{~cm}$ (Fig. 1D-F).

Treatment and therapy. A total of 65 patients underwent flexible ureteroscopic surgery to incise the renal cyst using the holmium laser or 1470-nm diode laser. The operation of 4 patients was terminated because the parapelvic cyst could not be located. A total of 3 patients undergoing holmium laser surgery were switched to 1470 -nm diode laser surgery due to excessive intraoperative bleeding. The data from 90 renal cysts were collected from 61 patients (Table I), including 43 renal cysts that received holmium laser surgery (holmium laser group) and 47 renal cysts that received 1470-nm diode laser surgery (1470-nm diode laser group).

Surgical procedure. A double-J stent was placed in the ureter in the two groups for 2 weeks preoperatively. After successful general anaesthesia, the access sheath of a flexible ureteroscope was advanced to the renal pelvis following the guidewire. A Flexible Video Ureteroscope (Olympus URF-V, F8.5/9.9) was placed into the renal pelvis through the access sheath. Preoperative CT with intraoperative ultrasonography identified the suspicious location of renal cysts. A translucent blue area was observed on the mucosa of the renal pelvis and calyx, which was considered the area of renal cysts adjacent to the renal pelvis and calyx. Ultrasound images revealed a smoke-like image in the cyst during the laser incision of the cyst wall. A $200-\mu \mathrm{m}$ holmium laser fibre $(1.0 \mathrm{~J}, 30 \mathrm{~Hz})$ was used. The power of the laser was $30 \mathrm{~W}(1.0 \mathrm{~J}, 30 \mathrm{~Hz})$, and the incision width of the cyst wall was $1-3 \mathrm{~cm}$ in the holmium laser group and 1470-nm diode laser group (Fig. 2A and B) to ensure that the cyst was completely connected to the renal pelvis. The flexible ureteroscope was advanced into the cyst to exclude tumour lesions. The proximal end of an F6 double-J stent was placed in the cyst under ultrasound guidance routinely following surgery. The catheter was removed 1-3 days post-surgery, and the F6 double-J stent was removed under a cystoscope 14-28 days later.

Follow up and evaluation. The success rate of cyst incision, operation time, intraoperative bleeding rate of the cyst wall, postoperative infection rate and surgical efficacy were compared between the two groups. Routine blood counts, renal function, routine urinalysis and urine culture were re-examined routinely after surgery. Plain CT scanning was used to evaluate the change in renal cysts, perirenal effusion and the position of double-J stenting 3-5 days after surgery. Renal CT was performed in the preoperative period (Fig. 2C), 1 month after surgery (Fig. 2D) and 6 months after surgery (Fig. 2E).

The following variables were included in the current study: Age, sex, side of cyst (unilateral or bilateral), cyst size, location (upper pole, medium pole and inferior pole), simple cyst/polycystic, Bosniak I/II score, blood loss, pre- and postoperative cystic diameter, diameter of cyst incision and complications (such as the presence of hematoma in renal cyst, fever condition and Lumbago) during and after surgery. The primary endpoint was efficacy of the treatment. Secondary endpoints were safety, pain and the resolution of other complications (including hematoma in renal cyst, fever condition and hematuria). All patients underwent radiological imaging of the kidneys using repeated CT 1 and 6 months after surgery.

A nurse in the Department of Urology evaluated pre- and postoperative lumbar pain scores of the patients. The pain score was assessed using a 10-point visual analogue scale ranging from 0 (no pain) to 10 (severe pain). Pain intensity was graded on a scale of 0 to 3 (slight), 4 to 6 (moderate) and $>6$ (severe) (10-11). Patients with a flank pain score $>3$, or with a residual pain rating, were categorized as symptomatic lumbago. Patients with pain scores $<3$ were recorded as being asymptomatic. 

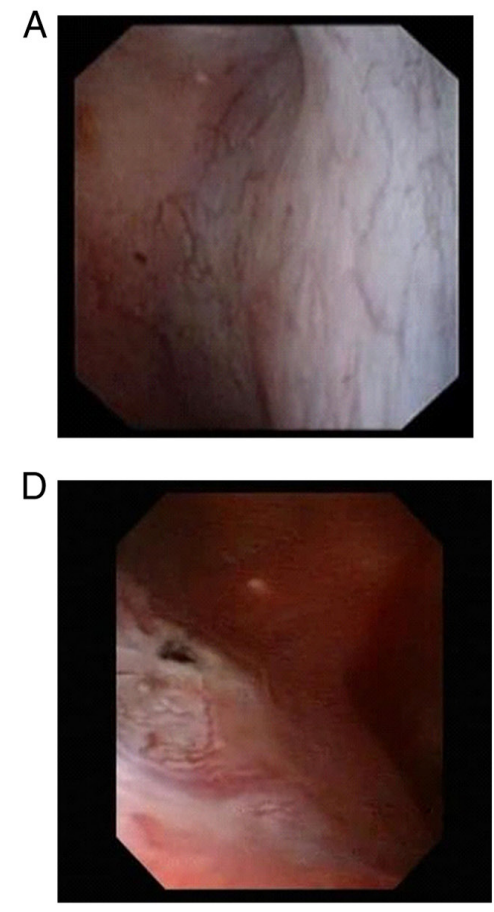

B

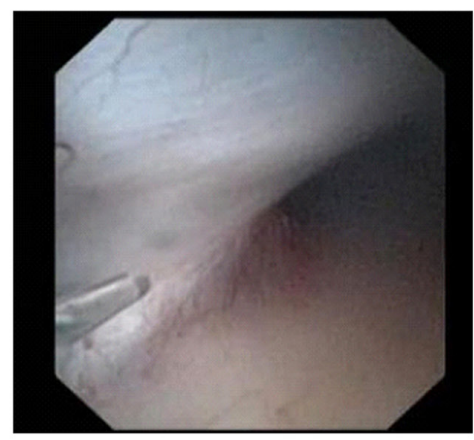

E

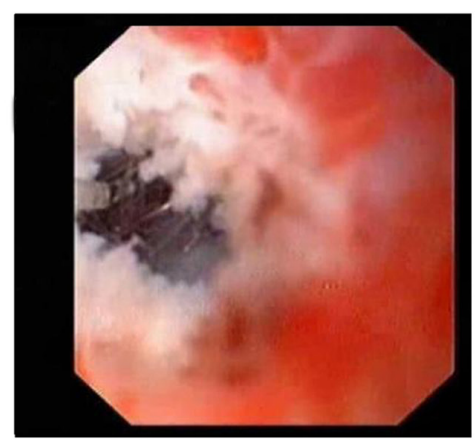

C

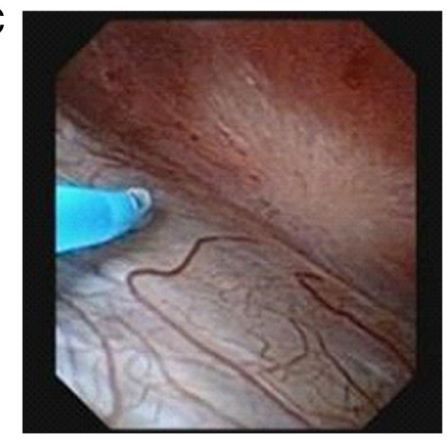

F

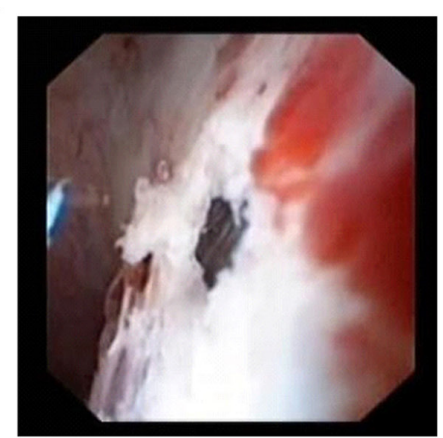

Figure 1. Images of cysts in 6 representative patients. (A-C) Endoscopic appearance of thin-walled cysts. (D-F) Endoscopic appearance of thick-walled cysts.

A

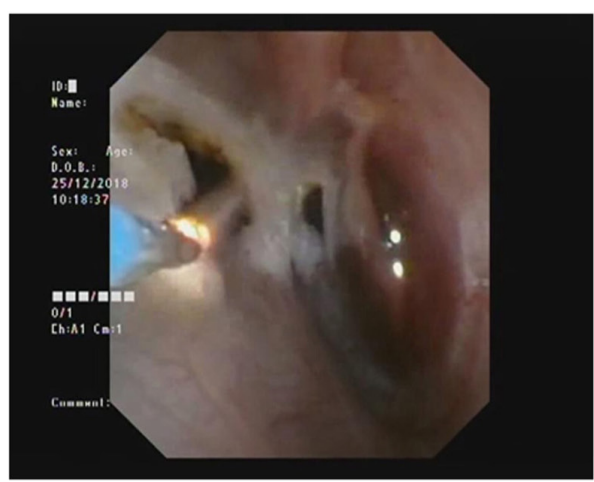

B

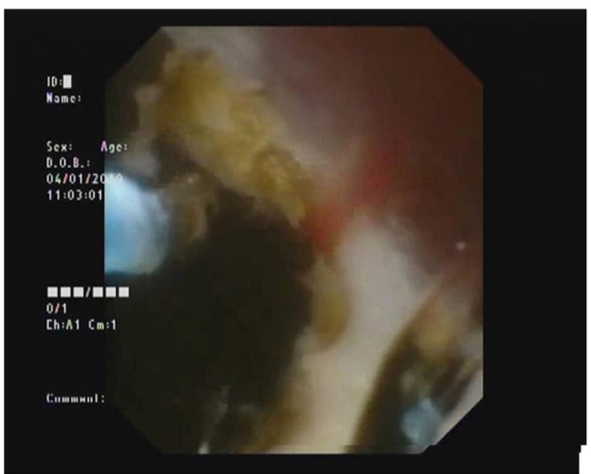

C

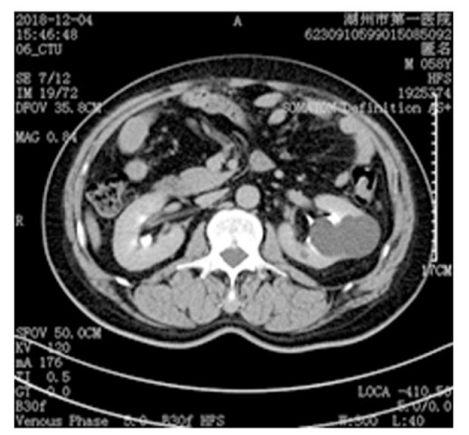

\section{D}

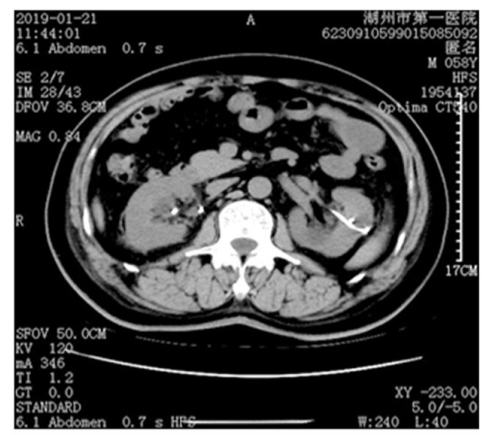

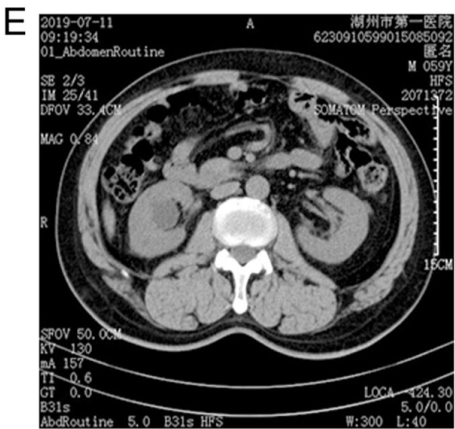

Figure 2. Intraoperative images and postoperative clinical efficacy of 1470-nm diode laser on parapelvic thick-walled renal cysts. (A) Intraoperative incision of parapelvic thick-walled renal cysts using a 1470-nm diode laser in the different patients. (B) Haemostatic effect of the 1470-nm diode laser on thick parapelvic renal cyst wall. CT scan of a representative patient with thick parapelvic renal cyst wall in the (C) preoperative period, (D) 1 month after 1470-nm diode laser surgery and (E) 6 months after 1470-nm diode laser surgery in the same patient.

Statistical analysis. SPSS 19.0 software (IBM Corp.) was used for statistical analyses. Measurement data are expressed as means \pm SD. Paired t-test was used to compare the pre- and post-operative curative effects in the same group. Independent sample t-test and Mann-Whitney U test was used to compare the pre- and post-operative curative effects between the two groups. Quantitative data are expressed as numbers (percentage), and the $\chi^{2}$ test or Fisher's test was used for comparison between these groups. $\mathrm{P}<0.05$ was considered to indicate a statistically significant difference. 
Table I. Patient characteristics between the 1470-nm and holmium laser group.

\begin{tabular}{|c|c|c|c|}
\hline Parameters & $\begin{array}{c}\text { 1470-nm } \\
\text { laser } \\
(\mathrm{n}=47)\end{array}$ & $\begin{array}{l}\text { Holmium } \\
\text { laser } \\
(\mathrm{n}=43)\end{array}$ & P-value \\
\hline Sex & & & 0.718 \\
\hline Male & 28 & 24 & \\
\hline Female & 19 & 19 & \\
\hline Age (years) & $60(50,64)^{\mathrm{a}}$ & $59(45,63)^{\mathrm{a}}$ & 0.531 \\
\hline Unilateral/bilateral cyst & & & 0.771 \\
\hline Unilateral & 27 & 26 & \\
\hline Bilateral & 20 & 17 & \\
\hline Simple cysts/polycystic & & & 0.324 \\
\hline Simple cysts & 31 & 24 & \\
\hline Polycystic & 16 & 19 & \\
\hline Kidney Cyst position, n (\%) & & & 0.997 \\
\hline Renal pelvis & 22 & 20 & \\
\hline Superior pole & 9 & 9 & \\
\hline Medium pole & 9 & 8 & \\
\hline Inferior pole & 7 & 6 & \\
\hline Bosniak (I/II) & & & 0.313 \\
\hline I & 39 & 36 & \\
\hline II & 8 & 7 & \\
\hline Cyst diameter (cm) & $5.11 \pm 0.65$ & $5.14 \pm 0.83$ & 0.422 \\
\hline Combined disease, $\mathrm{n}(\%)$ & & & 0.974 \\
\hline Kidney stones & 13 & 11 & \\
\hline Localized calyceal water & 8 & 9 & \\
\hline Hypertension & 16 & 13 & \\
\hline Diabetes & 7 & 7 & \\
\hline Coronary heart disease & 7 & 5 & \\
\hline
\end{tabular}

${ }^{\mathrm{a} Q u a n t i l e ~ s p a c i n g ~ f o r ~ n o n-n o r m a l l y ~ d i s t r i b u t e d ~ d a t a . ~}$

\section{Results}

Characteristics of patients at baseline. A total of 65 patients underwent flexible ureteroscopy to cut renal cysts and drain cyst fluid using a holmium laser or 1470-nm diode laser. A total of 4 patients were excluded as the parapelvic renal cyst wall was not able to be located during surgery. Flexible ureteroscopic holmium laser surgery was switched to flexible ureteroscopic 1470-nm diode laser surgery in 3 patients due to intraoperative bleeding. Therefore, the clinical data of 90 independent renal cysts were collected from 61 patients ( 35 males and 26 females; Table I), including 43 renal cysts that received holmium laser surgery and 47 renal cysts that received 1470-nm diode laser surgery. There was no significant differences in the general characteristics between the 2 groups prior to surgery. A total of 24 cases of independent renal cysts with renal calculi $(0.5-1.5 \mathrm{~cm})$ received holmium laser lithotripsy before decortication and drainage of renal (data not shown).

Perioperative results. Flexible ureteroscopic decortication and drainage of parapelvic renal cysts were completed under general
Table II. Perioperative results and follow-up.

\begin{tabular}{lccc}
\hline & $\begin{array}{c}1470-\mathrm{nm} \\
\text { laser } \\
(\mathrm{n}=47)\end{array}$ & $\begin{array}{c}\text { Holmium } \\
\text { laser } \\
(\mathrm{n}=43)\end{array}$ & P-value \\
Parameters & 20 & 22 & 0.413 \\
\hline Wall thickness & 27 & 21 & \\
Thin & 47 & 43 & \\
Thick & $1.81 \pm 0.38$ & $1.72 \pm 0.52$ & 0.194 \\
Thin wall+thick wall & & & \\
Diameter of cyst & & & \\
incision (cm) & $5.11 \pm 0.65$ & $5.14 \pm 0.83$ & 0.422 \\
Cyst diameter & $1.59 \pm 0.64$ & $1.61 \pm 0.68$ & 0.417 \\
Preoperation (cm) & $1.1(0.7,1.4)$ & $1.2(0.9,1.9)$ & 0.110 \\
One month (cm) & 0 & 4 & 0.048 \\
Six months (cm) & 5 & 5 & 1.000 \\
Hematoma in renal cyst & 9 & 8 & 0.950 \\
Fever condition & & & \\
Lumbago & & & \\
Radiologic failure & 0 & 5 & 0.252 \\
At 1 month & 2 & & \\
At 6 months & & & \\
\hline
\end{tabular}

aPatients with a flank pain score $>3$, or with a residual pain rating.

anaesthesia in the two groups. There was no statistical difference in the incision diameter of the renal cyst during surgery $(1.81 \pm 0.38$ vs. $1.72 \pm 0.52 \mathrm{~cm} ; \mathrm{P}=0.194$; Table II). Notably, the incision diameter in the 1470-nm diode laser group significantly exceeded the holmium laser group in the thick-walled parapelvic renal cysts subgroup $[1.70(1.50,1.90)$ vs. $1.30(1.25,1.70) \mathrm{cm}$, $\mathrm{P}=0.007$; Table III].

The results at one and six months after surgery. CT was re-examined, and the double-J stenting was removed in the holmium laser group and the 1470-nm diode laser group 1 and 6 months after surgery. It was observed that in patients with renal cyst recurrence that required clinical reoperation, the cysts drained were not completely blocked or unstable at 1 month, and the cysts drained were blocked or stable at 3 months. The renal cystic diameter of the two groups separately was markedly reduced 1 and 6 months after surgery. However, there was no significant difference in the renal cystic diameter between the two groups 1 month after surgery $(1.59 \pm 0.64$ vs. $1.61 \pm 0.68 \mathrm{~cm} ; \mathrm{P}=0.422)$, and the diameter of the renal cyst had a lower trend in the 1470-nm diode laser group than the holmium laser group 6 months after surgery [1.1(0.7, 1.4) vs. $1.2(0.9,1.9) \mathrm{cm} ; \mathrm{P}=0.110]$. The cystic diameter 1 month after surgery did not exhibit any significant difference between thin and thick-walled cyst subgroups, but a marked increase was noted in the holmium laser group $(1.46 \pm 0.73$ vs. $1.78 \pm 0.59 \mathrm{~cm}$; $\mathrm{P}=0.064$; Table IV). In the thin-walled and thick-walled cysts subgroups that received the holmium laser (Table III), 1 case recurred in the thin-walled cysts subgroup of holmium laser treatment, and 5 cases recurred in the thick-walled cysts subgroup of holmium laser 6 months after surgery. No cases recurred in the thin-walled cysts subgroup of the 1470-nm 
Table III. The perioperative results in thin-walled cysts subgroups and thick-walled cysts subgroups of two lasers and follow-up.

\begin{tabular}{lccc} 
A, Thin wall & & & \\
\hline & $\begin{array}{c}1470-\mathrm{nm} \\
\text { laser } \\
(\mathrm{n}=47)\end{array}$ & $\begin{array}{c}\text { Holmium } \\
\text { laser } \\
(\mathrm{n}=43)\end{array}$ & P-value \\
Parameters & 20 & 22 & \\
\hline Number of cysts & 1.95 & 1.95 & 0.859 \\
Diameter of cyst & $(1.53,2.28)$ & $(1.5,2.28)$ & \\
incision (cm) & & & \\
Cyst diameter & $5.16 \pm 0.74$ & $5.18 \pm 0.89$ & 0.473 \\
Preoperation (cm) & $1.43 \pm 0.67$ & $1.46 \pm 0.73$ & 0.447 \\
One month (cm) & $1.01 \pm 0.38$ & $1.03 \pm 0.53$ & 0.454 \\
Six months (cm) & 0 & 1 & 1.000 \\
Hematoma in renal cyst & 0 & 1 & 1.000 \\
Fever condition & 4 & 4 & 1.000 \\
Lumbago & & & \\
Radiologic failure & 0 & 0 & - \\
At 1 month & 0 & 1 & 1.000 \\
At 6 months & & & \\
\hline
\end{tabular}

B, Thick wall

\begin{tabular}{lccc}
\hline & $\begin{array}{c}1470-\mathrm{nm} \\
\text { laser } \\
(\mathrm{n}=47)\end{array}$ & $\begin{array}{c}\text { Holmium } \\
\text { laser } \\
(\mathrm{n}=43)\end{array}$ & P-value \\
Parameters & 27 & 21 & \\
\hline Number of cysts & 1.70 & 1.30 & 0.007 \\
Diameter of cyst & $(1.50,1.90)$ & $(1.25,1.70)$ & \\
incision (cm) & & & \\
Cyst diameter & $5.07 \pm 0.59$ & $5.10 \pm 0.79$ & 0.441 \\
Preoperation (cm) & $1.70 \pm 0.60$ & $1.78 \pm 0.59$ & 0.332 \\
One month (cm) & $1.21 \pm 0.57$ & $1.88 \pm 0.94$ & 0.002 \\
Six months (cm) & 0 & 3 & 0.077 \\
Hematoma in renal cyst & 5 & 4 & 1.000 \\
Fever condition & 5 & 4 & 1.000 \\
Lumbago & & & \\
Radiologic failure & 0 & 0 & - \\
At 1 month & 2 & 5 & 0.252 \\
At 6 months & & & \\
\hline
\end{tabular}

diode laser, and only 2 cases recurred in the thick-walled cysts subgroup of the 1470-nm diode laser 6 months after surgery. The diameter of the renal cyst was significantly lower in the thin-walled cysts subgroup that received the holmium laser than the thick-walled cysts subgroup that received the holmium laser at 6 months post-surgery $(1.03 \pm 0.53$ vs. $1.88 \pm 0.94 \mathrm{~cm}$; $\mathrm{P}=0.0004$; Table IV).

No difference in cystic diameter was indicated between the two subgroups that received the 1470-nm diode laser at the six-month check-up (thin-walled vs. thick-walled, $1.01 \pm 0.38$ vs. $1.21 \pm 0.57 \mathrm{~cm}$, respectively; $\mathrm{P}=0.155$; Table IV).
The diameter of the renal cyst was decreased in the 1470-nm diode laser group compared with the holmium laser group 6 months after surgery $[1.1(0.7,1.4)$ vs. $1.2(0.9,1.9) \mathrm{cm} ; \mathrm{P}=0.110$; Table II], and no significant difference in cyst diameter was observed between the thin-walled cysts subgroups treated with the two lasers 6 months after surgery $(1.01 \pm 0.38$ vs. $1.03 \pm 0.53 \mathrm{~cm}$, $\mathrm{P}=0.454$; Table III).

\section{Discussion}

Laparoscopic surgery for renal cysts was first described in 1992 , and this technique is now widely used for treatment (12). Antegrade percutaneous ureteroscopic unroofing has also examined in the treatment of renal cysts in recent years (13). Compared with exogenous renal cysts, parapelvic renal cysts are more likely to cause symptoms, such as bleeding and obstruction of the renal collecting system, which urgently require treatment by urologists (1). It is often difficult for urologists to treat parapelvic cysts using laparoscopic surgery or percutaneous ureteroscopic surgery since the cysts may have proximity to the renal hilar structures and pelvis (14). The surgical path of flexible ureteroscopic surgery runs closer to parapelvic renal cysts than the laparoscopic surgery anatomically (14). As this technique is transluminal naturally and leads to less bleeding, less risk and faster recovery, it has been favoured by a considerable number of urologists $(2,14-22)$. The key to the incision and drainage of parapelvic renal cysts via flexible ureteroscopy is the accurate location of the cysts. The present study suggested that the evaluation of cyst positioning should include ultrasonography, intravenous urography, retrograde pyelography, CT and an enhanced CT (2,14-20). A recent Chinese randomized controlled trial confirmed that the injection of methylene blue into the parapelvic renal cysts reduced the identification time and operation time of the cysts under a flexible ureteroscope (21). A previous study revealed that MPR-CTU combined with a precise intraoperative ultrasonography-guided flexible ureteroscope exerted better therapeutic effects (increased the successful rate of surgery and reduced complications) via easily locating the cystic wall compared with the flexible ureteroscope technique (5).

Holmium lasers are commonly used for the incision and drainage of parapelvic renal cysts under a flexible ureteroscope $(5,14,22)$. Yu et al (14) used a holmium laser to incise and drain endogenous symptomatic renal cystic diseases under a flexible ureteroscope. During the 24-month postoperative follow-up period, no cysts were detected in 26 patients with parapelvic cysts, and no serious perioperative complications occurred. A study by Luo et al (16) used a 200-mm laser fibre to treat renal parapelvic cysts using a flexible ureteroscope. Within the 14 cases used in the aforementioned study, cyst size was reduced by $50 \%$ of the previous size in only 1 case, cyst sizes were reduced to $<50 \%$ of the previous size in 4 cases and no cysts were detected in the other 10 cases, after a mean follow-up of 44 \pm 17.24 months (range 24-84). None of the patients exhibited intraoperative complications. A number of previous studies have used the $200 \mathrm{~nm}$ fiber setting $(14,16,17,21,23)$. Therefore, the $200 \mathrm{~nm}$ fiber was used at the hospital in the current study. Future studies should compare the two different therapeutic effects of different settings (365 vs. $200 \mathrm{~nm}$ ). During the 6 months of follow-up in the present study, it was revealed that the recurrence rate was higher in the thick-walled cysts subgroup 
Table IV. The perioperative cyst diameter in thin-walled cysts subgroups and thick-walled cysts subgroups of two lasers and follow-up.

\begin{tabular}{llcr}
\hline Parameters & Treatment groups & $\begin{array}{c}\text { Thin wall subgroup } \\
\text { diameter }(\mathrm{cm})\end{array}$ & $\begin{array}{c}\text { Thick wall subgroup } \\
\text { diameter }(\mathrm{cm})\end{array}$ \\
\hline A, One month & & & \\
\hline Cyst characteristics & 1470-nm laser $(\mathrm{n}=20)$ & $1.43 \pm 0.67$ & $1.70 \pm 0.60$ \\
Cyst characteristics & Holmium laser $(\mathrm{n}=22)$ & $1.46 \pm 0.73$ & $1.78 \pm 0.59$ \\
\hline
\end{tabular}

B, Six months

\begin{tabular}{lccc}
\hline Parameters & Treatment groups & $\begin{array}{c}\text { Thin wall subgroup } \\
\text { diameter }(\mathrm{cm})\end{array}$ & $\begin{array}{c}\text { Thick wall subgroup } \\
\text { diameter }(\mathrm{cm})\end{array}$ \\
\hline Cyst characteristics & 1470-nm laser $(\mathrm{n}=27)$ & $1.01 \pm 0.38$ & $1.21 \pm 0.57$ \\
Cyst characteristics & Holmium laser $(\mathrm{n}=21)$ & $1.03 \pm 0.53$ & $1.88 \pm 0.94$ \\
\hline
\end{tabular}

Data are presented as mean $\pm \mathrm{SD}$.

with holmium laser incision, and the diameter of thin-walled renal cysts was significantly lower than thick-walled renal cysts $(1.03 \pm 0.53$ vs. $1.88 \pm 0.94 \mathrm{~cm} ; \mathrm{P}=0.0004$; Table IV). The present study demonstrated that the holmium laser was effective for haemostasis of thin-walled renal cysts, but it was much less effective for the haemostasis of thick-walled renal cysts under a flexible ureteroscope. Moreover, there was a significant difference observed between thick/thin walled values combined and the two treatment techniques $(\mathrm{P}=0.048$; Table II), indicating that the $1470 \mathrm{~nm}$ laser was more effective for haemostasis overall. The current study also observed that the diameter of the holmium laser incision of the thin-walled renal cyst was 1.95(1.5,2.28; quantile spacing for non-normally distributed data) $\mathrm{cm}$, and the diameter of thick-walled renal cysts was 1.30(1.25,1.70; quantile spacing for non-normally distributed data) $\mathrm{cm}$. It may be suggested that the surgery is less favourable due to intraoperative bleeding, which may decrease the long-term curative effect due to the reduced incision diameter of cysts.

The 1470-nm diode laser treatment apparatus was a semiconductor laser, which possesses higher power and provides a continuous infrared laser. The 1470-nm laser produces little bleeding and exhibits a good curative effect $(7,24)$. The 1470-nm diode laser is widely used in the treatment of benign prostatic hyperplasia. To the best of our knowledge, the current study was the first to use the 1470-nm diode laser in the treatment of parapelvic renal cysts. According to biological and physical principles, the higher cell and tissue absorption of the laser reduces the penetration depth. Compared with the holmium laser, the 1470-nm diode laser is more easily absorbed by haemoglobin and water, which allows heat to be concentrated in a small piece of tissue with a penetration depth of 2-3 $\mathrm{mm}$ (7). This characteristic may reduce intraoperative bleeding, which was conducive to expanding the diameter of the cyst incision, draining of the cystic fluid and improving the surgical effect.

The results of the present study indicated that the incision diameter of thick-walled parapelvic renal cysts in the 1470-nm diode laser group was significantly higher compared with the holmium laser group in the perioperative period. The current study concluded that the 1470-nm diode laser was significantly superior to the holmium laser in haemostasis, as the risk of bleeding was reduced, and the incision diameter of parapelvic renal cysts was expanded to facilitate the drainage of cystic fluid. The 1470-nm diode laser and the holmium laser exerted equivalent effects on thick-walled and thin-walled renal cysts 1 month after surgery. It may be suggested that the placement of double-J stenting may serve an important role in draining cystic fluid and preventing cystic atresia within 1 month following surgery. In the current study, it was observed that in patients with renal cyst recurrence that required clinical reoperation, the cysts drained were not completely blocked or unstable at 1 month, and the cysts drained were blocked or stable at 3 months. The surgical effect of the 1470-nm diode laser was comparable to the holmium laser in the thin-walled parapelvic renal cysts subgroup 6 months after surgery. Therefore, the current study concluded that the thin-walled renal cyst was not prone to atresia or relapse after the incision. However, the surgical effect of 1470-nm diode laser was superior to the holmium laser in the thick-walled parapelvic renal cysts subgroup 6 months after surgery. Due to the excellent haemostatic effect of the 1470-nm diode laser, the surgeon could enlarge the incision diameter of thick-walled parapelvic renal cysts as much as possible, which made the cyst less prone to atresia and facilitated cystic fluid drainage. These results indicated that renal cysts should be open-incised as extensively as possible (2). However, the results of the present study also indicated that the haemostatic effect of the holmium laser was poor on thick-walled parapelvic renal cysts in the clinical procedure. The surgeon reduced the incision diameter of thick-walled parapelvic renal cysts out of caution in the holmium laser group, which made the cysts prone to atresia and poor drainage of cystic fluid and may have led to cyst recurrence.

In the current study, the 1470-nm diode laser was used to treat parapelvic renal cysts under a flexible ureteroscope. Compared with the holmium laser, the 1470-nm diode laser exhibited a number of advantages in the treatment of thick-walled parapelvic renal cysts, and may be used as an alternative choice for the treatment of thick-walled parapelvic renal cysts. However, the 
1470-nm diode laser was not the optimal treatment in thin-walled cysts since it is not significantly superior to holmium laser.

\section{Acknowledgements}

Not applicable.

\section{Funding}

This manuscriptordocument was supported by theNatural Science Foundation of Zhejiang Province (grant nos. LGF18H050001 and LY17H050004), National Natural Science Foundation of China, (grant no. 81370799), Zhejiang Provincial Medical and Health Science and Technology Plan Project (platform focus; grant no. 2016 ZDB 012), and Huzhou Municipal Science and Technology Bureau (grant no. 2016GY23).

\section{Availability of data and materials}

The datasets used and/or analyzed during the current study are available from the corresponding author on reasonable request.

\section{Authors' contributions}

YC contributed to experiment implementation, data analysis, figure composing and manuscript writing. RW and XS performed data analysis and manuscript editing. JT, JS, ZF and ZS were involved with data collection. XJ was involved in project design and manuscript writing. All authors read and approved the final manuscript.

\section{Ethics approval and consent to participate}

All clinical experiments and laboratory research were performed according to protocols that were approved by the ethical committee the First Affiliated Hospital of Huzhou Teacher's University, and each patient participated after providing written informed consent.

\section{Patient consent for publication}

Not applicable.

\section{Competing interests}

The authors declare that they have no competing interests.

\section{References}

1. Umemoto Y, Okamura T, Akita H, Yasui T and Kohri K: Clinical evaluation of parapelvic renal cysts: Do these represent latent urological malignant disease? Asian Pac J Cancer Prev 10: 1119-1120, 2009.

2. Basiri A, Hosseini SR, Tousi VN and Sichani MM: Ureteroscopic management of symptomatic, simple parapelvic renal cyst. J Endourol 24: 537-540, 2010.

3. Kutcher R, Amodio JB and Rosenblatt R: Uremic renal cystic disease: Value of sonographic screening. Radiology 147: 833-835, 1983.

4. Taguchi K, Harper JD, Stoller ML, Duty BD, Sorensen MD, Sur RL, Usawachintachit M, Tzou DT, Wenzler DL, Isaacson D, et al: Identifying factors associated with need for flexible ureteroscope repair: A Western Endourology STone (WEST) research consortium prospective cohort study. Urolithiasis 46: 559-566, 2018.
5. Wang R, Wang N, Tang J, Chen Y and Gao J: The safety and efficacy of MPR-CTU combined with precise intraoperative ultrasonography guided flexible ureteroscope in the treatment of renal cystic disease. Exp Ther Med 15: 283-287, 2018.

6. Camargo AHLA, Cooperberg MR, Ershoff BD, Rubenstein JN, Meng MV and Stoller ML: Laparoscopic management of peripelvic renal cysts: University of California, San Francisco, experience and review of literature. Urology 65: 882-887, 2005.

7. Zhao Y, Liu C, Zhou G, Yu C, Zhang Y and Ouyang Y: A retrospective evaluation of benign prostatic hyperplasia treatment by transurethral vaporization using a $1470 \mathrm{~nm}$ laser. Photomed Laser Surg 31: 626-629, 2013.

8. Liu Z, Zhao Y, Wang X, Song M and Shi B: Critical reviews of 1470-nm laser vaporization on benign prostatic hyperplasia. Lasers Med Sci 33: 323-327, 2018.

9. Silverman SG, Pedrosa I, Ellis JH, Hindman NM, Schieda N, Smith AD, Remer EM, Shinagare AB, Curci NE, Raman SS, et al: Bosniak classification of cystic renal masses, version 2019: An update proposal and needs assessment. Radiology 292: 475-488, 2019.

10. Breivik EK and Skoglund LA: Comparison of present pain intensity assessments on horizontally and vertically oriented visual analogue scales. Methods Find Exp Clin Pharmacol 20: 719-724, 1998.

11. de Jong AE, Bremer M, Schouten M, Tuinebreijer WE and Faber AW: Reliability and validity of the pain observation scale for young children and the visual analogue scale in children with burns. Burns 31: 198-204, 2005.

12. Yoder BM and Wolf JS Jr: Long-term outcome of laparoscopic decortication of peripheral and peripelvic renal and adrenal cysts. J Urol 171: 583-587, 2004.

13. Hu J, Dirie NI, Yang J, Xia D, Lu Y, Yu X and Wang S: Percutaneous ureteroscopy laser unroofing-a minimally invasive approach for renal cyst treatment. Sci Rep 7: 14445, 2017.

14. Yu W, Zhang D, He X, Zhang Y, Liao G, Deng G and Jin B: Flexible ureteroscopic management of symptomatic renal cystic diseases. J Surg Res 196: 118-123, 2015.

15. Li EC, Hou JQ, Yang LB, Yuan HX, Hang LH, Alagirisamy KK, Li DP and Wang XP: Pure natural orifice translumenal endoscopic surgery management of simple renal cysts: 2-year follow-up results. J Endourol 25: 75-80, 2011.

16. Luo Q, Zhang X, Chen H, Liu Z, Chen X, Dai Y and Zhao Z: Treatment of renal parapelvic cysts with a flexible ureteroscope. Int Urol Nephrol 46: 1903-1908, 2014.

17. Mao X, Xu G, Wu H and Xiao J: Ureteroscopic management of asymptomatic and symptomatic simple parapelvic renal cysts. BMC Urol 15: 48, 2015.

18. Mancini V, Cormio L, d'Altilia N, Benedetto G, Ferrarese P, Balzarro M, Defidio L and Carrieri G: Retrograde intrarenal surgery for symptomatic renal sinus cysts: Long-term results and literature review. Urol Int 101: 150-155, 2018.

19. Liaconis H, Pautler SE and Razvi HA: Ureteroscopic decompression of an unusual uroepithelial cyst using the holmium: YAG laser. J Endourol 15: 295-297, 2001.

20. Zhao Q, Huang S, Li Q, Xu L, Wei X, Huang S, Li S and Liu Z: Treatment of parapelvic cyst by internal drainage technology using ureteroscope and holmium laser. West Indian Med J 64: 230-235, 2015.

21. Wang Z, Zeng X, Chen C, Wang T, Chen R and Liu J: Methylene blue injection via percutaneous renal cyst puncture used in flexible ureteroscope for treatment of parapelvic cysts: A modified method for easily locating cystic wall. Urology 125 : 243-247, 2019.

22. Shen J, Chen Y and Wang R: Efficacy and complication of flexible ureteroscopic holmium laser incision for simple renal cysts: A retrospective study. J Endourol 33: 881-886, 2019.

23. Wen J, Xu G, He G, Wang B, Mao X and Zhang S: The clinical efficacy and safety of flexible ureteroscopic treatment for parapelvic renal cyst and secondary renal stone. Urol J 17: 243-247, 2020

24. Te AE, Malloy TR, Stein BS, Ulchaker JC, Nseyo UO, Hai MA and Malek RS: Photoselective vaporization of the prostate for the treatment of benign prostatic hyperplasia: 12-month results from the first United States multicenter prospective trial. J Urol 172: 1404-1408, 2004. International (CC BY-NC-ND 4.0) License. 